\title{
National health service healthcare staff experience and practices regarding complementary and alternative medicine: an online survey
}

\begin{abstract}
The use of complementary and alternative medicine (CAM) is increasing. The most common reported reason for CAM use is dissatisfaction with conventional healthcare. Several studies have reported factors influencing CAM usage and beliefs in the general public but the beliefs of healthcare staff are less well known. This paper reports the results of an online survey of 537 healthcare staff. Our study demonstrated an increased rate of patient referral for CAM from both personal CAM users and those trained in CAM. There was a high level of optimism amongst respondents as to the role CAM may play in patient care with mental health, depression and palliative care cited as the areas with highest expected benefit. Doctors were generally less optimistic about the likelihood of benefit compared to other healthcare staff. Implications for clinical practice, future research and staff education are discussed.
\end{abstract}

Keywords: healthcare, professionals, staff, cam, survey, personal use
Volume 5 Issue 4 - 2017

\author{
Jenny Jones,' Suzanne Rayner, ${ }^{2}$ Shona Logue, ${ }^{2}$ \\ Elizabeth Imray, ${ }^{3}$ Derek Stewart, ${ }^{4}$ Stephen J \\ Leslie 2,5 \\ 'Faculty of Health Science, Plymouth University, UK \\ ${ }^{2}$ Department of Medicine, Raigmore Hospital, UK \\ ${ }^{3}$ Culloden Medical Practice, UK \\ ${ }^{4}$ Robert Gordon University, UK \\ ${ }^{5}$ Department of Diabetes \& Cardiovascular Science, University \\ of the Highlands and Islands, UK
}

Correspondence: Stephen J Leslie, Highland Heartbeat
Centre, Cardiac Unit, Raigmore Hospital, Inverness, IV2 3UJ, UK,
Tel 44 I 463 705459, Email Stephen.leslie@nhs.net

Received: January 22, 2017 | Published: February 16, 2017

\section{Introduction}

The use of complementary and alternative medicine (CAM) is increasing, although rates of CAM use reported in the literature vary widely $(10 \%-86 \%) \cdot{ }^{1-6}$ In the UK, the average lifetime prevalence of CAM use is over $50 \%$, with herbal medicine the most popular, followed by homeopathy, aromatherapy, massage and reflexology. ${ }^{1}$ Being female, younger (aged $<65$ years) ${ }^{2}$ and having a chronic condition ${ }^{3}$ are associated CAM use. The most common reported reason for use of CAM is dissatisfaction with conventional healthcare. ${ }^{4}$

According to the National Centre for Complementary and Integrative Health, complementary medicine is defined as 'a nonmainstream practice used together with conventional medicine' and alternative medicine defined as 'a non-mainstream practice used in place of conventional medicine'. ${ }^{7}$ CAM encompasses a wide variety of health-related philosophical approaches to disease, often based on frameworks that have little in common with the scientific principles of conventional medicine. There is no uniform definition of what constitutes a CAM practice (whether practitioner delivered or selfadministered) and range from psychosocial interventions to notions of 'energies' that are non-biological in nature and have no known measurable biological correspondence, ${ }^{5}$ while others rely on plant or animal-derived chemical treatments, from well-defined molecular preparations in common herbal remedies or unregulated supplements to homeopathic ultra-molecular diluted remedies. ${ }^{5}$ Fewer than $7 \%$ of any CAM practices offer scientific evidence for specific benefits. ${ }^{6}$

Despite the lack of robust evidence, clinical staff are advised to discuss CAM with patients where appropriate, to encourage patient disclosure of use in order to adjust conventional treatment and give contra-indication advice where necessary. ${ }^{8,9}$ The Scottish Executive Health Department has advised Scottish health boards that they should take into account the growing public interest in CAM, when planning future service provision. ${ }^{10}$ As most CAM lacks formal scientific evidence, some research suggests that NHS staff rely on their own personal views or perceptions if asked for informed opinions or when integrating CAM into practice. ${ }^{11-13}$
To date, there has been no survey across all healthcare staff addressing the extent of personal CAM use in staff and the perceived outcome of patient CAM referral, with previous studies often focusing on medical and nursing staff. This is significant as other healthcare professionals and healthcare support workers often spend significant amounts of time with patients and their knowledge and beliefs surrounding CAM have potential to provide a significant influence on patients' perceptions and usage.

\section{This study aimed to answer the following research questions:}

i. Does staff personal usage of CAM influence 'referral' of patients for CAM therapy?

ii. Does previous staff CAM training (and delivery) influence referral of patients for CAM therapy?

iii. Do staff perceive that CAM has a potential role to play in the management of patient symptoms, and in which conditions?

For the purpose of these research questions and from this point on 'referral' is taken as referral for, or suggested patient use of, CAM.

\section{Methods}

\section{Subjects and setting}

This was an e-mail survey, sent to all healthcare staff with potential for access to patients (6985) listed in the internal e-mail system, in a single NHS health board in the north of Scotland (NHS Highland) in January 2014. The NHS Highland Associate Medical Director approved the use of the NHS Highland staff email address list and one email invitation to complete the survey was sent.

\section{Questionnaire development}

Due to the lack of a validated tool to answer the research questions, a 22 question bespoke survey was designed and developed in several iterative stages, with testing in small samples to ensure face validity until the final survey design was reached. Specific CAM modalities 
mentioned in the survey were taken from the House of Lords Science and Technology Committee seminal Sixth Report "Complementary and Alternative Medicine" (2000). ${ }^{5}$ Since 2007, all NHS Scotland staff has access to NHS mail accounts ${ }^{14}$ and we were granted access to the e-mail address of all healthcare staff in one health board area. A web-based survey format offered the advantage of lower cost and higher and more immediate response capacity in comparison to postal administration. ${ }^{15}$

\section{Data collection}

The sent e-mail described the survey research aim, assured anonymity of responses and included an embedded hyperlink to the Bristol Online Survey service (http://www.survey.bris.ac.uk). The survey website data collection was not restricted to one response per computer as many NHS staff share computer access. Data were collected as multiple choice check box response (only one answer allowed), multiple choice check box response (multiple answers allowed) and basic textual demographic data (e.g. job title and grade). At the end of the study period the survey results were downloaded from the Bristol Online Survey website into Excel (Microsoft Excel Version 10, Microsoft, Redmond, Washington, USA) for further analysis.

\section{Data analysis and statistics}

Staff respondents were categorised depending on their roles into 'nurse' (all grades), 'medical' (e.g. doctor or dentist (all grades)), 'healthcare professional (HCP)' (e.g. physiotherapist, occupational therapist, clinical scientist, pharmacist, speech and language therapist, radiographer, addiction workers, podiatrist, CBT therapists) and 'healthcare support worker (HCSW)' (e.g. auxiliary and administrative staff). We performed a Pearson's chi squared test on categorical data where appropriate with statistical significance taken at $\mathrm{p}<0.05$.

\section{Ethics}

Appropriate approval was gained to use the NHS Highland staff email address. No formal ethical approval was required for this staff survey and this was confirmed by NHS Highland Research and Development office.

\section{Results}

Of those contacted (6985), $541(7.7 \%)$ staff responded on or before the 3 -week cut-off response date.

\section{Demographics}

The majority of respondents, 225 (42.1\%), were nursing staff, with remaining respondents falling into the following distribution: 139 (26.0\%) medical, 137 (25.6\%) HCP and 24 (4.5\%) HCSW. This represented the following approximate response rate per subgroup: $6.2 \%$ nursing staff, $21.1 \%$ medical, $15.6 \% \mathrm{HCP}$ and $1.3 \%$ HCSW. 270 $(50.5 \%)$ respondents worked in a hospital setting.

\section{Personal use of CAM and referral}

The majority, 457 (85.4\%) of respondents had personally used CAM, demonstrating at least $4.7 \%$ of current healthcare staff use CAM. In those who used CAM, the majority, 402 (88.0\%) found the outcomes of personal CAM use either 'all' or 'mostly' positive, with HCPs expressing the most positive views (Table 1).

HCSW were excluded from the table due to small numbers.

Table I Perceived outcome following personal CAM use

Outcome following personal use of CAM $(\%(\mathbf{N}))$
all positive
mostly positive
neutral
mostly negative
all negative

Of all respondents, 284 (53.1\%) were own CAM users and referred patients for CAM as opposed to $26(4.9 \%)$ of non-CAM users who referred patients. Overall, there was an association between own use of CAM and referral of patients CHI 22.7 with OR 3.28 (95\% CI 1.98 to $5.45, \mathrm{p}<0.001)$. Subgroup analysis of referral rates showed significant associations between own use and CAM referral in nursing staff OR $5.61(95 \%$ CI 2.00 to $15.71, \mathrm{p}=0.001)$ and medical staff OR $4.01(95 \%$ CI 1.87 to $8.60, \mathrm{p}<0.001)$. HCP and HCSW could not be analysed due to small expected frequencies $(<5)$ in one or more categories.

\section{CAM training/ delivery and referral}

The majority of respondents either 'agreed' or 'strongly agreed' that more information about CAM risks and benefits should be taught to health care professionals (Table 2). Less than a quarter, 121 (22.6\%), of participants had received self-reported training in CAM delivery. Of all staff who had CAM training, there was a higher percentage onward referral rate for CAM therapy compared with those that did not have CAM training, 94 (77.7\%) vs 216 (52.2\%) OR 3.19 (95\% CI 2.00 to $5.10, \mathrm{p}<0.001)$.

HCSW were excluded from the table due to small numbers.

\begin{tabular}{llll} 
All (457) & HCP (225) & Medical (94) & Nurses (202) \\
\hline $30.2(138)$ & $17.8(40)$ & $17.0(16)$ & $34.7(70)$ \\
$57.8(264)$ & $78.2(176)$ & $57.4(54)$ & $57.4(116)$ \\
$7.7(35)$ & $2.2(5)$ & $16.0(15)$ & $6.9(14)$ \\
$2.0(9)$ & $1.3(3)$ & $4.3(4)$ & $1.0(2)$ \\
$1.5(7)$ & $0.4(1)$ & $5.3(5)$ & $0.0(0)$
\end{tabular}

\section{NHS staff perceptions of efficacy and usefulness of CAM for patient use}

Just over half of the respondents had personally referred or recommended patients for CAM, $301(56.0 \%)$. In those who referred or recommended CAM, 208 (76.5\%) reported the patient outcomes as either 'all' or 'mostly' positive, with little difference between staff groups. There was a broad perception throughout staff groups that CAM could have a potential role in the management of patient symptoms in several chronic illnesses, ranging 59.6-88.6\% of positive responses. Mental health, depression and palliative care were cited amongst the areas with highest expected benefit with heart failure, chronic obstructive pulmonary disease and asthma the lowest.

\section{Discussion}

This is the first online survey distributed to all healthcare staff in one Scottish Health Board (NHS Highland) addressing personal and patient CAM usage and referral practice. Previous studies have focused on medical and nursing staff. ${ }^{11,12,16}$

The survey found that the majority of respondents acknowledged using CAM and most reported positive outcomes from CAM use. This 
number represents at least 1 in $20(\sim 5 \%)$ of the healthcare staff. Half of all respondents had personally referred patients for CAM, although only $22 \%$ had received any formal training in any CAM modality. Furthermore, the majority of respondents, particularly nursing staff, expressed the view that CAM may have a potential role to play in the management of patients' symptoms in common longer-term chronic/illnesses/conditions. This indicates that personal views or perceptions largely guide current decision-making in patient referrals and perceived usefulness of CAM in healthcare.

Previous studies have similarly evaluated personal attitudes and referral rates in healthcare staff. A survey examining Aberdeen Royal Infirmary nurses' views and self-use of CAM found that $80 \%$ were own CAM users and $74 \%$ would recommend to others. $93 \%$ of nurses surveyed had not had any formal education on CAM. ${ }^{12}$ A separate survey focusing on the use of CAM in obstetric practice in North East Scotland showed $32.5 \%$ of the healthcare professionals involved in care during pregnancy (obstetricians, midwives and anaesthetists) would recommend CAM to patients, with recommendation 8 times more likely if the healthcare professional was within the $53.8 \%$ of personal CAM user. ${ }^{11}$ In comparison to this study, we found a higher rate of referral (56\%) and of own CAM use (85.4\%). Overall odds ratio was positive for referral amongst personal CAM use from both our survey and the North East Scotland study. ${ }^{11}$

Our survey confirmed that many NHS staff express a desire for more information on CAM risks and benefits and believe that healthcare professionals should receive more training in relation to CAM. At present, CAM education does not formally appear on undergraduate nursing, occupational therapy or pharmacy undergraduate curricula ${ }^{13,17}$ and although CAM education modules are offered in selected medical school curricula, the content is reported to be largely superficial and inconsistent. ${ }^{18,19}$ In a national survey of physiotherapists, just over half used CAM as part of their treatment of lower back pain, despite most therapists being unsure of its efficacy. ${ }^{20}$ These findings suggest that there is a gap in professional training for many clinical disciplines, which could have patient safety implications.

For example, herbal remedy use is often associated with the perception that herbal preparations have fewer side effects than prescription drugs. ${ }^{21}$ However, potentially serious adverse effects and treatment reactions have been reported between some herbal preparations or supplements and cardiovascular drugs ${ }^{22}$. Only around $40 \%$ of patients are thought to inform their primary care physician after CAM use $\mathrm{e}^{23,24}$ and evidence indicates that adverse reactions to CAM treatments may be under-reported to healthcare auth3rities. ${ }^{25}$ CAM use has also been linked with increased risk of non-compliance with conventional healthcare treatments. ${ }^{26,27}$ As doctors, nurses and allied healthcare staff may be reliant on internet-based anecdotal information rather than evidence-based journals to make informed decisions about of CAM when discussing the risks and benefits with patients, ${ }^{27}$ our findings indicate there is a clear need for more CAM education in professional training at all levels of multidisciplinary care delivery.

\section{Limitations}

This was a single centre study with a relatively low response rate, however this is in keeping with other on-line surveys. With over five hundred participants, our study is larger than many previous CAM studies and this also allowed evaluation between staff groups.

It is a logical assumption that respondents to a CAM survey are likely to have a greater interest in CAM than non-respondents and therefore at risk of selection bias. This makes difficult to estimate the actual number of healthcare staff involved in CAM. A systematic review in 2012 estimated $9.8-76 \%$ prevalence over 12month of usage of any CAM. As $85.4 \%$ of our respondents personally used CAM this is comparatively high and therefore indicates a likely biased sample due to self-selection. ${ }^{28}$

\section{Conclusion}

Despite a general lack of scientific evidence for CAM, our study demonstrated a high level of optimism as to the role CAM may play in patient care in the study respondents. Mental health, depression and palliative care were cited amongst the areas with highest expected benefit with heart failure, chronic obstructive airways disease and asthma the lowest. Doctors were generally less optimistic about the likelihood of benefit. Many staff expressed a need for more information and education on CAM risks and benefits, indicating unmet professional training needs.

\section{Acknowledgments}

None.

\section{Conflicts of interest}

Author declares there are no conflicts of interest.

\section{Funding}

None.

\section{References}

1. Zachezeweski.Improving flexibility. Physical Therapy. Philadelphia, USA: JB Lippincott Co; 1989.

2. Posadzki P, Watson LK, Alotaibi A, et al. Prevalence of use of complementary and alternative medicine (CAM) by patients/ consumers in the UK: systematic review of surveys. Clin Med (Lond). 2013;13(2):126-131.

3. Thomson P, Jones J, Evans JM, et al. Factors influencing the use of complementary and alternative medicine and whether patients inform their primary care physician. Complement Ther Med. 2011;20(1-2):4553.

4. Millar WJ. Patterns of use - alternative health care practitioners. Health Rep. 2001;13(1):9-21.

5. Ernst E. Homeopathic Galphimia for hay fever: A systematic review of randomised clinical trials and a critique of a published meta-analysis. Focus on Alternative and Complementary Therapies. 2011;16(3):200204

6. http://www.rebhp.org/articles/House.pdf

7. Ernst E. How much of CAM is based on research evidence? EvidenceBased Complementary and Alternative Medicine. (2011);1-3.

8. https://nccih.nih.gov/health/integrative-health\#cvsa

9. http://www.sign.ac.uk/pdf/sign125.pdf. Accessed on 25/06/2014.

10. http://www.sign.ac.uk/pdf/SIGN129.pdf

11. http://www.scot.nhs.uk/sehd/mels/HDL2005_37.pdf

12. Stewart D, Pallivalappila AR, Shetty A, et al. Healthcare professional views and experiences of complementary and alternative therapies in obstetric practice in North East Scotland: a prospective questionnaire survey. BJOG . 2014;121(8):1015-1019. 
13. Buchan S, Shakeel M, Trinidade A, et al. The use of complementary and alternative medicine by nurses. Br J Nurs. 2012;21(12):672-676.

14. Shorofi SA, Arbon P. Nurses' knowledge, attitudes, and professional use of complementary and alternative medicine (CAM): A survey at five metropolitan hospitals in Adelaide. Complement Ther Clin Pract. 2010;16(4):229-234.

15. http://www.connectingforhealth.nhs.uk/systemsandservices/nhsmail/ about/studies

16. Resnick, Robert M. Comparison of postal and online surveys: cost, speed, response rates and reliability. USA: Education Market Research and MCH Strategic Data; 2012.p.1-18.

17. Pallivalappila AR, Stewart D, Shetty A, et al. Complementary and Alternative Medicines Use during Pregnancy: A Systematic Review of Pregnant Women and Healthcare Professional Views and Experiences. 2013;205639.

18. Smith GD. The need for complementary and alternative medicine familiarisation in undergraduate nurse education. $J$ Clin Nurs. 2009;18(15):2113-2115.

19. Poynton L, Dowell A, Dew K, et al. General practitioners' attitudes toward (and use of) complementary and alternative medicine: A New Zealand nationwide survey. $N$ Z Med J. 2006;119(1247):1-10.

20. Brown J, Cooper E, Frankton L, et al. Complementary and alternative therapies: Survey of knowledge and attitudes of health professionals at a tertiary pediatric/women's care facility. Complement Ther Clin Pract. 2007;13(3):194-200.

21. Hughes CM, Quinn F, Baxter GD. Complementary and alternative medicine: Perception and use by physiotherapists in the management of lower back pain. Complement Ther Med. 12011;19(3):149-154.
22. Quan H, Lai D, Johnson D, et al. Complementary and alternative medicine use among Chinese and white Canadians. Can Fam Physician. 200/8;54(11):1563-1569.

23. Miller KL, Liebowitz RS, Newby LK. Complementary and alternative medicine in cardiovascular disease: A review of biologically based approaches. Am Heart J . 2004;147(3):401-411.

24. Thomson P, Jones J, Evans JM, et al. Factors influencing the use of complementary and alternative medicine and whether patients inform their primary care physician. Complement Ther Med. 2011;20(1$2): 45-53$.

25. MacLennan AH, Myers SP, Taylor AW. The continuing use of complementary and alternative medicine in South Australia: costs and beliefs in 2004. Med J Aust. 2006;184(1):27-31.

26. Braun LA, Tiralongo E, Wilkinson JM, et al. Adverse reactions to complementary medicine: The Australian Pharmacy experience. Int $J$ Pharm Pract. 2010;18(4):242-244.

27. Ernst E. Concompliance with conventional medicine and use of complementary/alternative medicine. J Postgrad Med. 2007;(2):85.

28. Jose VM, Bhalla A, Sharma N, et al. Study of association between use of complementary and alternative medicine and non-compliance with modern medicine in patients presenting to the emergency department. $J$ Postgrad Med. 2005;53(2):96-101.

29. Harris PE, Cooper KL, Relton C. Prevalence of complementary and alternative medicine (CAM) use by the general population: a systematic review and update. Int J Clin Pract. 2012;66(10):924-939. 